\title{
miR-940 inhibits cell proliferation and promotes apoptosis in esophageal squamous cell carcinoma cells and is associated with post-operative prognosis
}

\author{
HAIWEN WANG ${ }^{1}$, TAO SONG ${ }^{2}$, YANPING QIAO ${ }^{3}$ and JIANGTAO SUN ${ }^{4}$ \\ Departments of ${ }^{1}$ Cardio-Thoracic Surgery, ${ }^{2}$ Endocrinology, ${ }^{3}$ Hematology and ${ }^{4}$ Oncology, \\ Weihai Central Hospital, Weihai, Shandong 264400, P.R. China
}

Received October 12, 2018; Accepted August 30, 2019

DOI: $10.3892 /$ etm.2019.8279

\begin{abstract}
The present study aimed to examine microRNA (miR)-940 expression in esophageal squamous cell carcinoma (ESCC) tissues and cells, analyze its association with the clinicopathological features and prognosis of patients, and explore the potential underlying mechanisms. miR-940 expression in ESCC cell lines and a normal esophageal cell line was detected using reverse transcription-quantitative (RT-q)PCR. Furthermore, 210 resected ESCC tissue and para-carcinoma tissue specimens were collected, and miR-940 expression in those tissues was detected by RT-qPCR. In addition, the association of miR-940 with the clinicopathological features and prognosis of patients was analyzed. In an in vitro experiment, miR-940 mimics were transduced into ESCC cells by the liposome method. An MTT assay was used to detect the effect of miR-940 on the viability of ESCC cells. The influence of miR-940 on the cell cycle and apoptotic rate of ESCC cells was detected by flow cytometry. The present results indicated that the expression levels of miR-940 in human ESCC tissues and cell lines were markedly downregulated, and that low expression of miR-940 in ESCC tissues was significantly associated with a poor degree of differentiation, positive lymph node metastasis and advanced clinical stage. Kaplan-Meier survival analysis suggested that low miR-940 expression was associated with poor prognosis. Cox regression analysis revealed that lymph node metastasis, clinical stage and miR-940 expression were independent risk factors affecting the prognosis of patients. Overexpression of miR-940 in ESCC cells markedly reduced the cell viability, blocked the cell cycle at G0/G1 phase and promoted cell apoptosis. These results suggest that miR-940 is downregulated in ESCC,
\end{abstract}

Correspondence to: Dr Jiangtao Sun, Department of Oncology, Weihai Central Hospital, 3 West of Mi Shan East Road, Wendeng, Weihai, Shandong 264400, P.R. China

E-mail: sunjiangtaozlk@yeah.net

Key words: esophageal tumor, squamous cell carcinoma, microRNA-940, cell proliferation, cell cycle, apoptosis which is linked to the occurrence and progression of ESCC. Conversely, overexpression of miR-940 reduced the cell viability and promoted apoptosis of ESCC cells. Therefore, miR-940 may be a promising novel prognostic marker and anti-cancer target in ESCC.

\section{Introduction}

Esophageal carcinoma is one of the most common tumor types with a high grade of malignancy. Esophageal adenocarcinoma is common in European and American countries, while esophageal squamous cell carcinoma (ESCC) frequently occurs in Asian countries, including China (1). Esophageal carcinoma is mostly diagnosed at the advanced stage. In spite of the continuous improvement of treatments, including surgery and radiochemotherapy, the 5-year survival rate of esophageal carcinoma patients remains dismal due to delayed diagnosis and high recurrence rate $(2,3)$. Furthermore, the etiology and pathogenesis of esophageal carcinoma have remained to be fully elucidated, and effective molecular markers and targets for targeted therapy are currently lacking. Therefore, illustration of the molecular mechanisms of the genesis and metastasis of esophageal carcinoma, discovery of genes for specific and effective molecular targeted therapy and development of effective methods for early diagnosis, precise evaluation and prognostication are of great significance.

microRNAs (miRNAs/miRs) are a class of endogenous and highly conserved single-stranded non-coding small RNAs of 19-22 nucleotides in length. They are able to inhibit target gene expression at the post-transcriptional level through specifically binding with the 3'-untranslated region (3'-UTR) of their target mRNAs, thus exerting biological functions $(4,5)$. Studies have verified that miRNAs have important regulatory roles in cell proliferation, apoptosis, development and apoptosis, processes that are also closely associated with tumor pathogenesis, development and prognosis $(6,7)$. miR-940 is an important member of the miRNA family, which has been reported to be abnormally expressed in tumorous tissues and to have an important regulatory role $(8,9)$. However, to the best of our knowledge, miR-940 expression in ESCC has not been previously reported, and its association with prognosis remains elusive. 
In the present study, miR-940 expression was assessed in ESCC cell lines with various degrees of differentiation, as well as in a normal esophageal cell line, and in ESCC tissues and para-carcinoma tissues by using reverse transcription-quantitative (RT-q)PCR. The association of miR-940 expression with the clinicopathological features and prognosis in ESCC patients was analyzed. The possible mechanistic roles of miR-940 in the occurrence and development of ESCC were also investigated.

\section{Materials and methods}

Tissue specimens and clinical data. A total of 210 fresh surgically resected ESCC tissues and matched para-carcinoma tissues (at least $5 \mathrm{~cm}$ away from the tumor margin; 150 males and 60 females; aged 52-79 years old) were collected at Weihai Central Hospital (Weihai, China) between January 2011 and January 2012. Following excision, the tissue specimens were washed in saline within $30 \mathrm{~min}$, placed in liquid nitrogen and preserved in a refrigerator at $-80^{\circ} \mathrm{C}$. None of the patients had received any anti-tumor therapies, including radiotherapy, chemotherapy or biological immunotherapy; furthermore, they were not complicated by any primary tumors in other organs. All specimens were confirmed as squamous cell carcinoma by pathologists after surgery. A total of 52 cases received adjuvant radiotherapy and chemotherapy after the operation. The clinical features of the patients are presented in Table I. Their clinicopathological stage and criteria of tumor differentiation were evaluated according to the TNM classification system of the American Joint Committee on Cancer-International Union Against Cancer staging manual (10). The criteria for determining the differentiation of tumors are as follows: i) Well-dif ferentiated-prominent keratinization with pearl formation and a minor component of non-keratinizing basal-like cells, tumor cells arranged in sheets and low mitotic counts; ii) moderately differentiated-variable histologic features ranging from parakeratotic to poorly keratinizing lesions and pearl formation generally absent; iii) poorly differentiated-predominantly consisting of basal-like cells forming large and small nests with frequent central necrosis and with the nests consisting of sheets or pavement-like arrangements of tumor cells that are occasionally punctuated by small numbers of parakeratotic or keratinizing cells. Overall survival (OS) time was defined as the date of surgery to the date of death or completion of follow-up. Completion of follow-up, loss to follow-up and death of other causes were treated as the endpoint data. All patients had provided written informed consent to participate in the study. The present study was approved by the Medical Ethics Committee of Weihai Central Hospital (Weihai, China).

Cell culture and cell transfection. The human ESCC cell lines KYSE410 (poorly differentiated invasive ESCC, TE-13 (poorly differentiated), ECA-109 (moderately differentiated) and KYSE-510 (highly differentiated), as well as the normal human esophageal cell line Het-1A, were purchased from the Shanghai Cell Institute of the Chinese Academy of Sciences. In addition, the TE-13 cell line used in the present study was authenticated by STR profiling. All cells were placed in RPMI-1640 medium (Gibco; Thermo Fisher Scientific, Inc.) containing $10 \%$ fetal bovine serum (Invitrogen; Thermo
Fisher Scientific, Inc.) and cultured in an incubator containing $5 \% \mathrm{CO}_{2}$ at $37^{\circ} \mathrm{C}$. KYSE410 and TE-13 cells in the logarithmic growth phase were harvested and transfected with either miR-940 mimics (cat. no. B02002; Shanghai GenePharma Co., Ltd.) and miR-negative control (NC) (cat. no. B1012; Shanghai GenePharma Co., Ltd.), in strict accordance with the manufacturer's protocol for the Lipofectamine ${ }^{\mathrm{TM}} 2000$ kit (Invitrogen; Thermo Fisher Scientific, Inc.). At $24 \mathrm{~h}$ following transfection, miR-940 expression in cells was detected through RT-qPCR, so as to determine the efficiency of overexpression.

Total RNA extraction and RT-qPCR. Total RNA was extracted using a TRIzol kit (Invitrogen; Thermo Fisher Scientific, Inc.) according to the manufacturer's protocol and the purity of the extracted RNA was detected with an ultraviolet spectrophotometer. RNA with a 260/280 nm absorbance value ratio of 1.7-2.0 served as the qualification standard for subsequent experiments. Reverse transcription was performed immediately; alternatively, the RNA was stored in a refrigerator at $-80^{\circ} \mathrm{C}$. Reverse transcription of total RNA to complementary DNA was performed using a Moloney murine leukemia virus RT kit (Promega Corp.) according to the manufacturer's protocol. Specific primers for miR-940 and internal reference U6 were designed and synthesized by RiboBio. The primer sequences used were as follows: miR-940 forward, 5'-GTATAAAGGGCCCCC GCT-3' and reverse, 5'-AGGGTCCGAGGTATTCGCACT-3'; U6 forward, 5'-CATCACCATCAGGAGAGTCG-3' and reverse, 5'-TGACGCTTGCCCACAGCCTT-3'. qPCR was performed using the SYBR Green Realtime PCR Master Mix (Fermentas) and specific primers, with the complementary DNA used as the template. The LightCycle 96 fluorescent quantitative PCR machine (Roche) was used for the reaction and detection. The reaction conditions were as follows: 2 min at $94^{\circ} \mathrm{C}, 20 \mathrm{sec}$ at $94^{\circ} \mathrm{C}$ and $30 \mathrm{sec}$ at $60^{\circ} \mathrm{C}$ for 40 cycles. The relative expression of $\mathrm{miR}-940$ was calculated according to the $2^{-\Delta \Delta \mathrm{Cq}}$ method (11), with U6 used as the internal reference. All assays were performed in triplicate.

Cell viability detected by MTT assay. Cells transfected for $24 \mathrm{~h}$ were added to the 96-well cell culture plate (5,000 cells/well), and $20 \mu \mathrm{l}$ MTT (Sigma-Aldrich; Merck KGaA) was added to each well at 24, 48, 72 and $96 \mathrm{~h}$, followed by incubation for another $4 \mathrm{~h}$ at $37^{\circ} \mathrm{C}$. The supernatant was carefully aspirated, and subsequently, $150 \mu 1$ DMSO was added to each well, followed by agitation for $15 \mathrm{~min}$ to fully dissolve the crystals that had formed. The OD value was determined using a microplate reader (Bio-Tech Instruments) at a wavelength of $450 \mathrm{~nm}$. Three duplicate wells were set for each group.

Cell cycle analysis by flow cytometry. Cells transfected for $48 \mathrm{~h}$ were collected through digestion with $0.25 \%$ trypsin solution (EDTA-free) and washed with PBS for three times. The supernatant was discarded after centrifugation, and cells were re-suspended with $70 \%$ ethanol, in which they were fixed at $4^{\circ} \mathrm{C}$ for $18 \mathrm{~h}$. The cells were then collected through centrifugation $\left(1,000 \mathrm{x} \mathrm{g} ; 5 \mathrm{~min} ; 4^{\circ} \mathrm{C}\right)$ and washed with PBS twice, and the supernatant was discarded following centrifugation. Cells were then re-suspended with $400 \mu 1$ RNAse A (BD Biosciences)-containing propidium iodide (PI) staining 
Table I. Associations of miR-940 expression with clinicopathological characteristics of patients with esophageal squamous cell carcinoma.

\begin{tabular}{|c|c|c|c|c|}
\hline Clinicopathological feature & Cases (n) & miR-940 low $(n=100)$ & miR-940 high $(n=110)$ & P-value \\
\hline Sex & & & & 0.275 \\
\hline Male & 150 & $75(50.00)$ & $75(50.00)$ & \\
\hline Female & 60 & $25(41.67)$ & $35(58.33)$ & \\
\hline Age (years) & & & & 0.778 \\
\hline$>60$ & 162 & $78(48.15)$ & $84(51.85)$ & \\
\hline$\leq 60$ & 48 & $22(45.83)$ & $26(54.17)$ & \\
\hline Tumor size (cm) & & & & 0.179 \\
\hline$>5$ & 111 & $48(43.24)$ & $63(56.76)$ & \\
\hline$\leq 5$ & 99 & $52(52.53)$ & $47(47.47)$ & \\
\hline Tumor differentiation & & & & 0.034 \\
\hline Well/moderate & 125 & $52(41.60)$ & $73(58.40)$ & \\
\hline Poor & 85 & $48(56.47)$ & $37(43.53)$ & \\
\hline TNM stage & & & & 0.009 \\
\hline $\mathrm{I} / \mathrm{II}$ & 110 & $43(39.09)$ & $67(60.91)$ & \\
\hline III & 100 & $57(57.00)$ & $43(43.00)$ & \\
\hline Tumor location & & & & 0.588 \\
\hline Upper & 28 & $12(42.86)$ & $16(57.14)$ & \\
\hline Middle+lower & 182 & $88(48.35)$ & $94(51.65)$ & \\
\hline Lymphatic metastasis & & & & 0.001 \\
\hline Yes & 135 & $76(56.30)$ & $59(43.70)$ & \\
\hline No & 75 & $24(32.00)$ & $51(68.00)$ & \\
\hline
\end{tabular}

Values are expressed as n (\%). miR, microRNA.

solution, incubated in the dark at $4^{\circ} \mathrm{C}$ for $1 \mathrm{~h}$ and the cell cycle was detected using a flow cytometer.

Cell apoptosis detected by Annexin V-FITC/PI double-staining. After digestion with $0.25 \%$ trypsin solution (EDTA-free), cells transfected for $48 \mathrm{~h}$ were collected using centrifugation (111.8 x g; $\left.5 \mathrm{~min} ; 4^{\circ} \mathrm{C}\right)$. Binding buffer $(500 \mu \mathrm{l})$ was added to re-suspend the cells in accordance with the protocol of the Annexin V-FITC/PI double-staining cell apoptosis detection kit (Beyotime Institute of Biotechnology). Subsequently, $5 \mu \mathrm{l}$ Annexin V-FITC was added and the suspension was mixed sufficiently, followed by addition of $5 \mu \mathrm{l} \mathrm{PI}$, sufficient mixing and incubation in the dark at room temperature for 5-15 min. Finally, cell apoptosis was detected with a flow cytometer. The Annexin V-FITC ${ }^{+} \mathrm{PI}^{-}$cells were considered apoptotic and counted.

Statistical analysis. Data were analyzed using SPSS 20.0 statistical software (IBM Corp.). Measurement data were expressed as the mean \pm standard deviation and were compared using Student's t-test or one-way analysis of variance. A Student-Newman-Keuls test was used as a post-hoc test following ANOVA. The associations between miR-940 expression and various clinicopathological characteristics were assessed using a chi-square test. OS was assessed using the Kaplan-Meier method and groups were compared using the log-rank test. Univariate and multivariate analysis regarding
OS was performed using the Cox proportional hazards regression model. $\mathrm{P}<0.05$ was considered to indicate a statistically significant difference.

\section{Results}

miR-940 expression in ESCC tissues and cell lines. miR-940 expression in 210 pairs of ESCC tissues and matched para-carcinoma tissues was detected using RT-qPCR. As presented in Fig. 1A, compared with that in para-carcinoma tissues, miR-940 expression in ESCC tissues was markedly downregulated $(\mathrm{P}<0.001)$. Furthermore, miR-940 expression was analyzed in the human ESCC cell lines KYSE410, TE-13, ECA-109 and KYSE-510, as well as in the normal human esophageal cell line Het-1A. As presented in Fig. 1B, miR-940 expression in the human ESCC cell lines KYSE410, TE-13, ECA-109 and KYSE-510 was distinctly downregulated relative to that in the normal human esophageal cell line Het-1A $(\mathrm{P}<0.001)$, which was particularly obvious in the poorly-differentiated KYSE410 and TE-13 cell lines.

Association of miR-940 with clinicopathological parameters and prognosis in ESCC patients. To confirm the role of miR-940 expression in the development of ESCC, the associations of miR-940 with the clinicopathological parameters and prognosis of ESCC patients were then assessed. The ESCC 
Table II. Univariate and multivariate analysis of overall survival in 210 patients with ESCC.

Univariate analysis

Variables

Sex

(male vs. female)

$0.98(0.90 \sim 1.21)$

$1.19(0.94 \sim 1.51)$

0.148

(>60 vs. $\leq 60)$

$1.33(0.95 \sim 1.87)$

0.099

$(>5$ vs. $\leq 5)$

$2.88(1.23 \sim 6.75)$

0.015

$1.65(0.95 \sim 2.87)$

0.001

$2.87(1.30 \sim 6.33)$

0.076

TNM stage

(I/II vs. III)

$3.11(1.58 \sim 6.11)$

$1.25(0.94 \sim 1.66)$

0.124

(upper vs. female)

3.34 (1.82 6.13)

$<0.001$

$3.58(1.67 \sim 7.65)$

0.001

(no vs. yes)

$3.01(1.37 \sim 6.61)$

0.006

$2.44(1.14 \sim 5.20)$

0.021

miR, microRNA; ESCC, esophageal squamous cell carcinoma; RR, risk ratio.
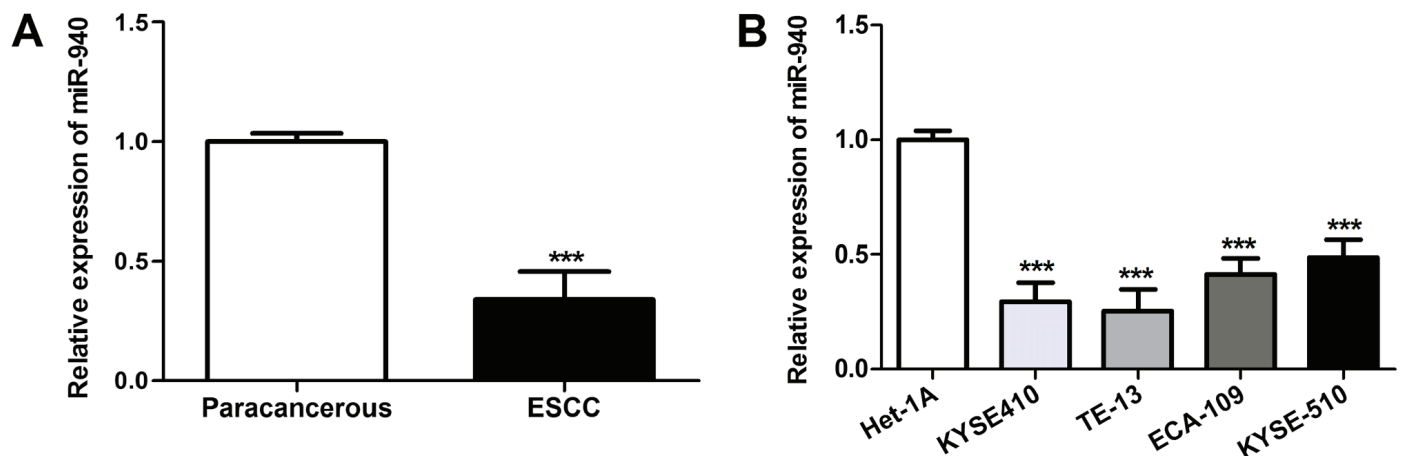

Figure 1. miR-940 is markedly downregulated in ESCC tissues and cell lines. (A) The relative expression of miR-940 in 210 pairs of ESCC tissues and para-carcinoma tissues was detected by RT-qPCR. (B) The relative expression of miR-940 in ESCC cell lines and a normal human esophageal cell line were detected by RT-qPCR. ${ }^{* * *}$ P $<0.001$ vs. control group. RT-qPCR, reverse transcription-quantitative PCR; ESCC, esophageal squamous cell carcinoma; miR, microRNA.

patients were divided into 2 subgroups according to their miR-940 expression status. The high miR-940 expression group included patients whose miR-940 level was higher than the average for all patients, while the low miR-940 expression group included patients whose miR-940 level was lower than the average for all patients. As indicated in Table I, low miR-940 expression was closely associated with poor tumor differentiation, positive lymph node metastasis and advanced clinical stage $(\mathrm{P}<0.05)$. Kaplan-Meier survival analysis suggested that the low miR-940 expression group had poor prognosis $(\mathrm{P}<0.01$; Fig. 2). Univariate Cox regression analysis was performed for all clinicopathological parameters (Table II). The tumor differentiation degree, lymph node metastasis, TNM stage and miR-940 expression were determined as risk factors affecting patient prognosis. Furthermore, risk factors with statistical

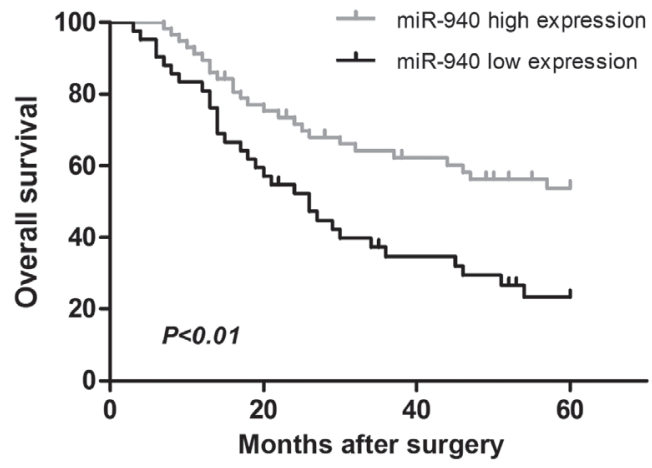

Figure 2. Low miR-940 expression is associated with poor prognosis Kaplan-Meier analysis was used to determine the probabilities of overall survival in patients with esophageal squamous cell carcinoma according to miR-940 expression. miR, microRNA. 

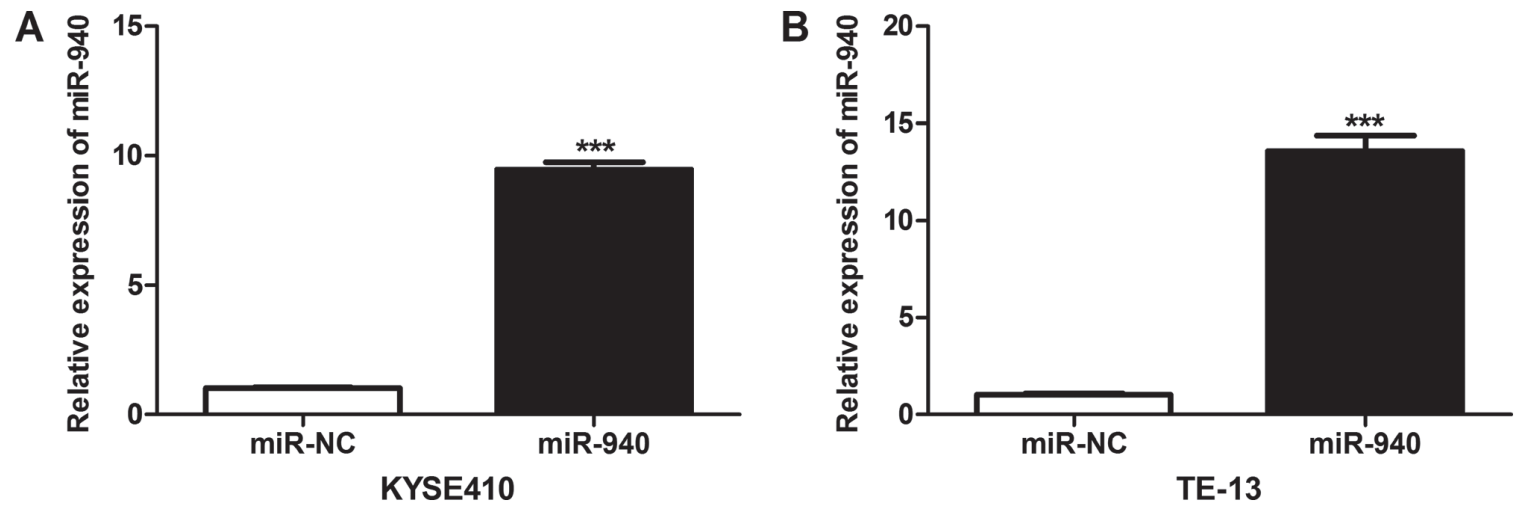

Figure 3. Confirmation of vector-mediated overexpression of miR-940 in esophageal squamous cell carcinoma cell lines. The relative expression of miR-940 was detected by reverse transcription-quantitative PCR in (A) KYSE410 and (B) TE-13 cells. ${ }^{* * *} \mathrm{P}<0.001$ vs. control group. Groups: miR-940, cells transfected with miR-940 mimics overexpression vector; miR-NC, cells transfected with negative control mimics vector. miR, microRNA.
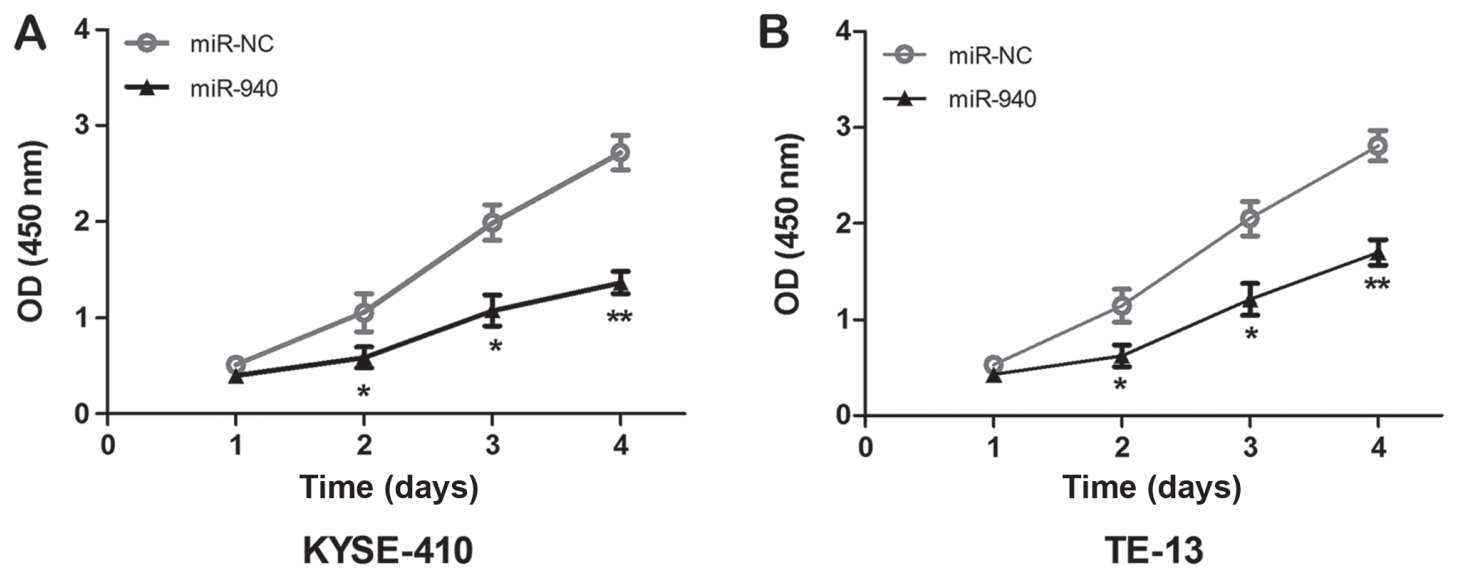

Figure 4. Overexpression of miR-940 in esophageal squamous cell carcinoma cells markedly reduces cell viability. MTT assays were used to determine the viability of (A) KYSE410 and (B) TE-13 cells. "P<0.05 and ${ }^{* * *} \mathrm{P}<0.01$ vs. control group. Groups: miR-940, cells transfected with miR-940 mimics overexpression vector; miR-NC, cells transfected with negative control mimics vector. miR, microRNA; OD, optical density.

significance were incorporated into the multivariate Cox regression model analysis. The results suggested that lymph node metastasis, TNM stage and miR-940 expression were independent risk factors affecting patient prognosis $(\mathrm{P}<0.05)$.

Effect of miR-940 overexpression on cell viability, cell cycle and apoptotic rate of ESCC cells. As presented in Fig. 3, RT-qPCR analysis confirmed that at $24 \mathrm{~h}$ after transfection, the miR-940 expression levels in KYSE410 and TE-13 cells in the miR-940 mimics group were markedly elevated $(\mathrm{P}<0.001)$, suggesting successful miR-940 mimics transfection and high efficiency of overexpression. The results of the MTT assay are presented in Fig. 4. It was observed that, compared with miR-NC group, the OD $450 \mathrm{~nm}$ values of KYSE410 and TE-13 cells transfected with miR-940 mimics were significantly decreased on days 2,3 and $4(\mathrm{P}<0.05)$, indicating that upregulation of miR-940 expression markedly suppressed the viability of ESCC cells. The cell cycle was detected by flow cytometry and the results are presented in Fig. 5. The G0/G1-phase population of KYSE410 and TE-13 cells with up-regulated miR-940 expression was significantly increased $(\mathrm{P}<0.01)$, while the proportions of cells in the $\mathrm{G} 2 / \mathrm{M}$ - and $\mathrm{S}$-phase were evidently decreased $(\mathrm{P}<0.05)$, indicating that overexpression of miR-940 caused cell cycle arrest of ESCC cells at the G0/G1 phase. The results of the flow cytometric analysis of apoptotic cells with Annexin V-FITC/PI double-staining are displayed in Fig. 6. The apoptotic rates of KYSE410 and TE-13 cells with overexpression of miR-940 were evidently elevated $(\mathrm{P}<0.01)$, indicating that miR-940 mimics promote apoptosis of ESCC cells.

\section{Discussion}

In spite of the progress made in the surgical and pharmacological therapy of esophageal carcinoma in recent years, the prognosis for esophageal carcinoma remains poor (12). Local recurrence and metastasis of esophageal carcinoma are major bottlenecks restricting the efficacy of esophageal carcinoma therapy and affecting the improvement of survival of esophageal carcinoma patients. To date, the mechanisms of the genesis and development of esophageal carcinoma have remained to be fully elucidated and no effective targeted therapeutics are available at present. Therefore, it is urgently required to illustrate the precise mechanisms of the genesis, development and metastasis of esophageal carcinoma at the molecular level and search for effective gene targets for targeted therapy of esophageal carcinoma. 
A

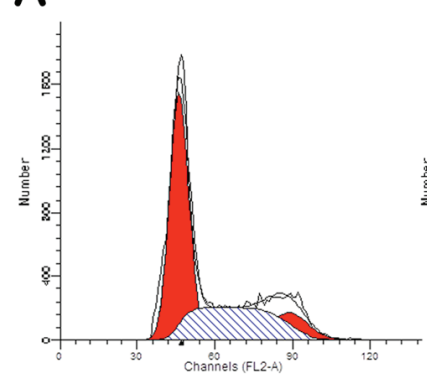

miR-NC
KYSE-410

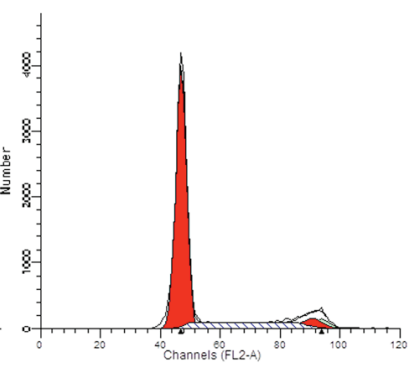

miR-940

KYSE-410

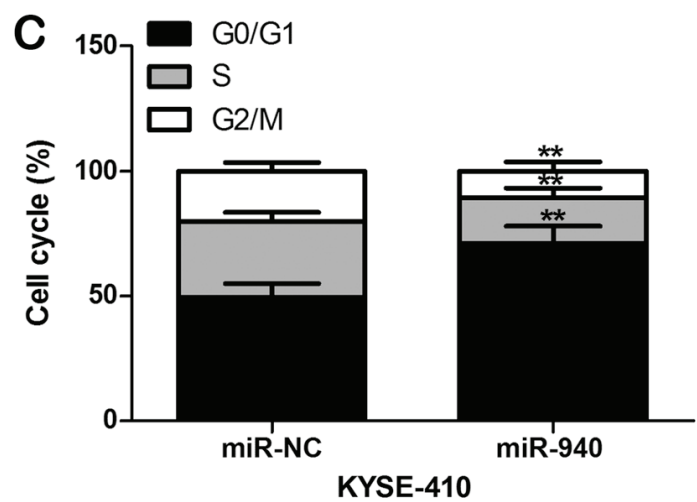

B

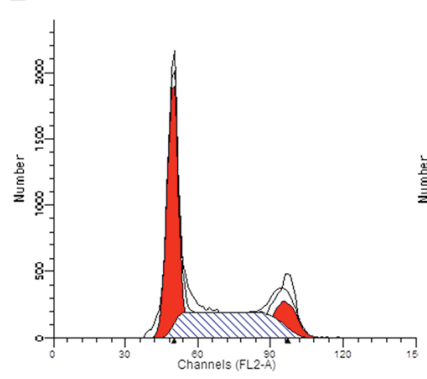

miR-NC
TE-13

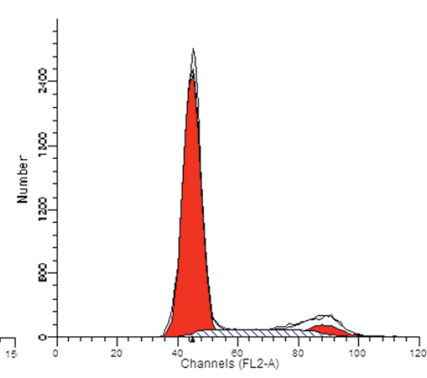

miR-940

TE-13

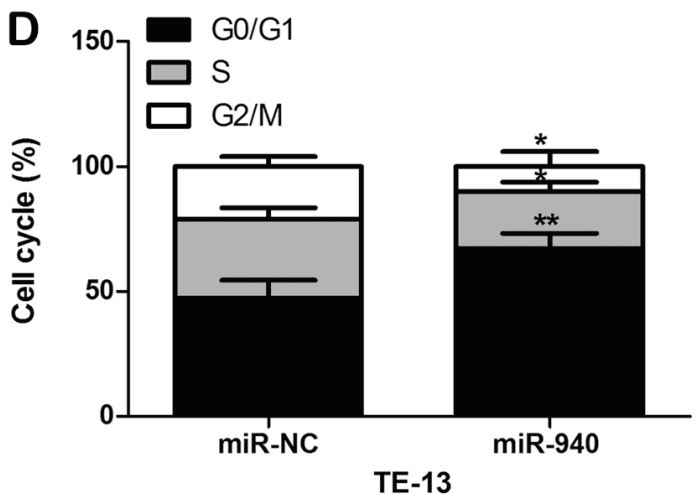

TE-13

Figure 5. Overexpression of miR-940 in esophageal squamous cell carcinoma cells causes cell-cycle arrest at the G0/G1 stage. (A and B) Cell cycle distribution of (A) KYSE410 and (B) TE-13 cells analyzed by flow cytometry. (C and D) Quantitative analysis of cell populations in different cell cycle phases for (C) KYSE410 and (D) TE-13 cells. " $\mathrm{P}<0.05$ and ${ }^{* *} \mathrm{P}<0.01$ vs. control group. Groups: miR-940, cells transfected with miR-940 mimics overexpression vector; miR-NC, cells transfected with negative control mimics vector. miR, microRNA.

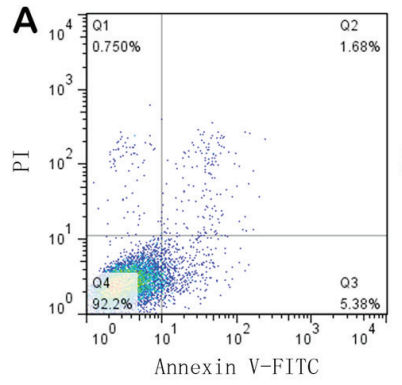

miR-NC

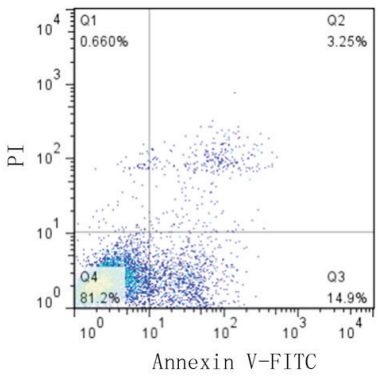

$\operatorname{miR}-940$

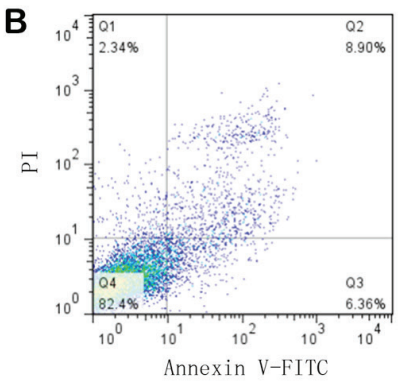

miR-NC

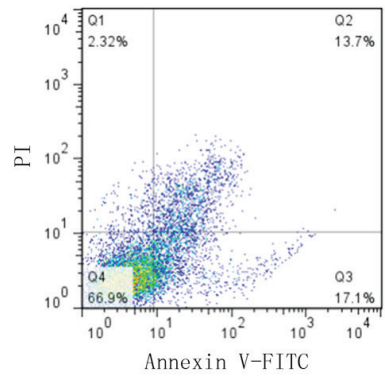

$\operatorname{miR}-940$

TE-13
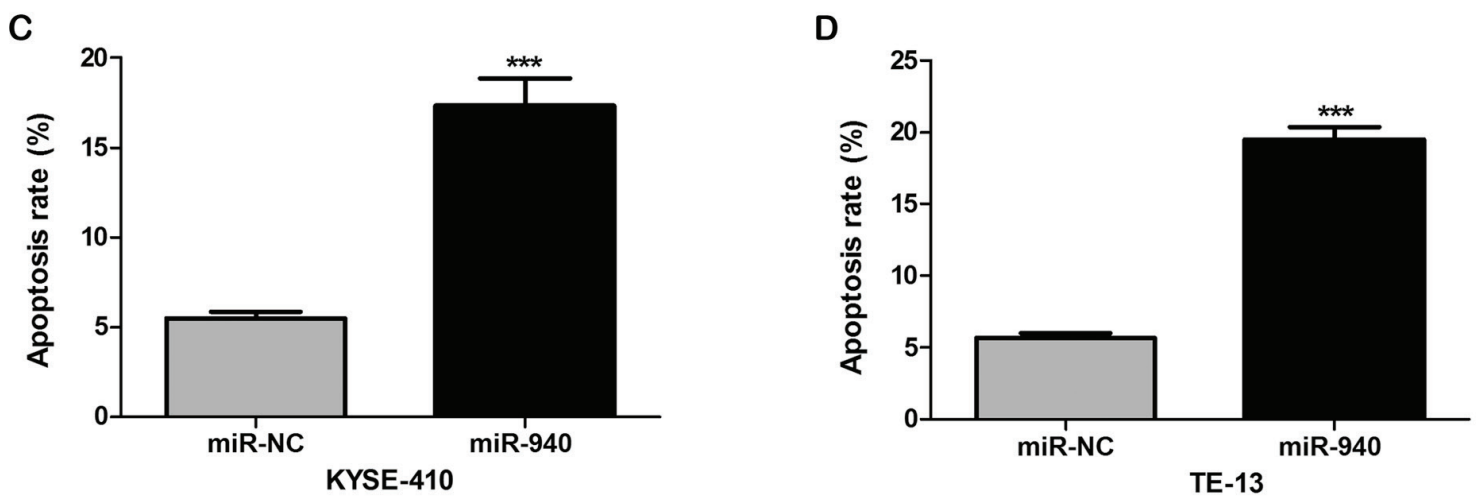

Figure 6. Overexpression of miR-940 in esophageal squamous cell carcinoma cells markedly promotes cell apoptosis. (A and B) Flow cytometric analysis of (A) KYSE410 and (B) TE-13 cells stained with Annexin V-FITC/PI. (C and D) Quantification of apoptosis in (C) KYSE410 and (D) TE-13 cells. **** P $<0.001$ vs. control group. Groups: miR-940, cells transfected with miR-940 mimics overexpression vector; miR-NC, cells transfected with negative control mimics vector. miR, microRNA; PI, propidium iodide; Q, quadrant. 
Aberrant expression of various miRNAs has been detected in multiple cancer types, which have crucial effects on tumor cell proliferation, apoptosis, invasion and migration. Therefore, differentially expressed miRNAs have become a hotspot in current research on tumors and gene therapy (13). Furthermore, numerous miRNAs have been verified to be involved in the genesis, development, invasion and metastasis of esophageal carcinoma $(14,15)$. miR-940 is one of the most important members of the miRNA family discovered recently. It has been indicated that miR-940 has an important role in various diseases. Liang et al (16) reported that downregulation of miR-940 had a key role in the development of tetralogy of Fallot in humans. Xu et al (17) indicated that serum miR-940 levels were significantly increased after exhaustive exercise in patients with congestive heart failure, which may be used as a marker for adaptive exercise in such patients. The expression pattern of miR-940 in malignant tumors is different from that in the above scenario. It has been reported that miR-940 expression is reduced in various types of malignant tumor, including ovarian cancer (9), prostate cancer (18), nasopharyngeal carcinoma (19) and hepatocellular carcinoma (20). Furthermore, overexpression of miR-940 may inhibit the proliferation, invasion and migration of tumor cells, suggesting that the role of miR-940 is that of a tumor suppressor gene in some malignant tumors $(9,18-20)$. Conversely, it has been indicated that the expression of miR-940 is increased in gastric cancer (21), bladder cancer (22) and pancreatic cancer (23), where it has the role of an oncogene. However, to date, miR-940 expression in ESCC has not been previously reported, to the best of our knowledge. The present study first reported on the abnormal expression of miR-940 in ESCC. The expression levels of miR-940 in 210 ESCC tissues and matched para-carcinoma tissues, as well as in the human ESCC cell lines KYSE410, TE-13, ECA-109 and KYSE-510, and in the normal human esophageal cell line Het-1A, were detected using RT-qPCR. The present results suggested that miR-940 expression in ESCC tissues and cells was decreased. Further statistical analysis indicated that low miR-940 expression was closely associated with poor tumor differentiation, positive lymph node metastasis and advanced clinical stage, suggesting that reduced miR-940 expression was closely linked to the development and metastasis of ESCC.

Studies on the association between miR-940 and prognosis for patients with malignant tumors are currently sparse. It has been reported that low miR-940 expression in hepatocellular carcinoma is associated with poor prognosis for patients (24). However, it has also been indicated that high expression of miR-940 is associated with poor prognosis in patients with gastric cancer (21). In the present study, post-operative follow-up was performed, the results of which indicated that patients in the low miR-940 expression group had a shorter OS and that low miR-940 levels are therefore associated with poor prognosis. Further multivariate analysis using the Cox regression model suggested that lymph node metastasis, clinical stage and miR-940 expression were independent risk factors affecting patient prognosis.

Excessive cell proliferation and blocked cell apoptosis are the basic biological characteristics of malignant tumor cells. To study the underlying mechanisms of action of miR-940 in ESCC, miR-940 mimics were transfected into ESCC cells to perform a gain-of-function experiment. The MTT assay suggested that miR-940 mimics suppressed the viability of ESCC cells. Cell cycle progression is a key factor regulating cell proliferation. The flow cytometric cell cycle analysis performed in the present study indicated that miR-940 mimics made the cells stay at G0 phase or blocked the cell cycle progression from G1- to S-phase. In addition, the proportion of cells in G0/G1 phase was markedly increased, suggesting that miR-940 suppresses the viability of ESCC cells partially through blocking the cell cycle. Finally, cell apoptosis was also detected through Annexin V-FITC/PI double-staining, and the results suggested that miR-940 mimics increased apoptosis of ESCC cells. Each miRNA has several regulatory target genes, and the final effects of a miRNA are the consequences of the comprehensive action of the complex downstream network of multiple genes. The precise targeted regulatory mechanisms by which miR-940 affects the biological characteristics of ESCC cells remain to be fully elucidated, which should be predicted by bioinformatics methods and assessed/verified through further cytological experiments.

In conclusion, the present study indicated that miR-940 expression is decreased in ESCC, and low miR-940 expression is significantly associated with poor tumor differentiation, advanced clinical stage, lymph node metastasis and poor prognosis. miR-940 is also an independent risk factor affecting patient prognosis. Overexpression of miR-940 in ESCC cells markedly reduced the cell viability, blocked the cell cycle at G0/G1-phase and promoted cell apoptosis. Therefore, miR-940 is a promising novel anti-cancer target and prognostic biomarker in ESCC.

\section{Acknowledgements}

Not applicable.

\section{Funding}

No funding was received.

\section{Availability of data and materials}

The datasets used and/or analyzed during the present study are available from the corresponding author on reasonable request.

\section{Authors' contributions}

JS designed the experiments. HW, TS and YQ performed the experiments. JS and HW analyzed the data. HW wrote the manuscript. All authors have read and approved the manuscript.

\section{Ethical approval and consent to participate}

The present study was approved by the Medical Ethics Committee of Weihai Central Hospital (Weihai, China). Informed consent was obtained from all patients.

\section{Patient consent for publication}

Not applicable. 


\section{Competing interests}

The authors declare that they have no competing interests.

\section{References}

1. Torre LA, Bray F, Siegel RL, Ferlay J, Lortet-Tieulent J and Jemal A: Global cancer statistics, 2012. CA Cancer J Clin 65: 87-108, 2015.

2. Chen W, Zheng R, Zhang S, Zeng H, Fan Y, Qiao Y and Zhou Q Esophageal cancer incidence and mortality in China, 2010 Thorac Cancer 5: 343-348, 2014.

3. Pennathur A, Gibson MK, Jobe BA and Luketich JD: Oesophageal carcinoma. Lancet 381: 400-412, 2013.

4. Shen J, Stass SA and Jiang F: MicroRNAs as potential biomarkers in human solid tumors. Cancer Lett 329: 125-136, 2013.

5. Sorel O and Dewals BG: MicroRNAs in large herpesvirus DNA genomes: Recent advances. Biomol Concepts 7: 229-239, 2016.

6. Mitchell PS, Parkin RK, Kroh EM, Fritz BR, Wyman SK, Pogosova-Agadjanyan EL, Peterson A, Noteboom J, O'Briant KC, Allen A, et al: Circulating microRNAs as stable blood-based markers for cancer detection. Proc Natl Acad Sci USA 105 10513-10518, 2008.

7. Paulmurugan R: MicroRNAs-a new generation molecular targets for treating cellular diseases. Theranostics 3: 927-929, 2013.

8. Rashed MH, Kanlikilicer P, Rodriguez-Aguayo C, Pichler M, Bayraktar R, Bayraktar E, Ivan C, Filant J, Silva A, Aslan B, et al: Exosomal miR-940 maintains SRC-mediated oncogenic activity in cancer cells: A possible role for exosomal disposal of tumor suppressor miRNAs. Oncotarget 8: 20145-20164,2017.

9. Wang F, Wang Z, Gu X and Cui J: miR-940 upregulation suppresses cell proliferation and induces apoptosis by targeting PKC- $\delta$ in ovarian cancer OVCAR3 cells. Oncol Res 25: 107-114, 2017.

10. Rice TW, Ishwaran H, Ferguson MK, Blackstone EH and Goldstraw P: Cancer of the esophagus and esophagogastric junction: An eighth edition staging primer. J Thorac Oncol 12: 36-42, 2017.

11. Livak KJ and Schimittgen TD: Analysis of relative gene expression data using real-time quantitative PCR and the 2(-Delta Delta C(T)) method. Methods 25: 402-408,2001.

12. Ohashi S, Miyamoto S, Kikuchi O, Goto T, Amanuma Y and Muto M: Recent advances from basic and clinical studies of esophageal squamous cell carcinoma. Gastroenterology 149: $1700-1715,2015$

13. Pereira DM, Rodrigues PM, Borralho PM and Rodrigues CM: Delivering the promise of miRNA cancer therapeutics. Drug Discov Today 18: 282-289, 2013
14. Harada K, Baba Y, Ishimoto T, Shigaki H, Kosumi K, Yoshida N, Watanabe $\mathrm{M}$ and Baba $\mathrm{H}$ : The role of microRNA in esophageal squamous cell carcinoma. J Gastroenterol 51: 520-530, 2016.

15. Feber A, Xi L, Luketich JD, Pennathur A, Landreneau RJ, Wu M, Swanson SJ, Godfrey TE and Litle VR: MicroRNA expression profiles of esophageal cancer. J Thorac Cardiovasc Surg 135: 255-260, 2008.

16. Liang D, Xu X, Deng F, Feng J, Zhang H, Liu Y, Zhang Y, Pan L, Liu Y, Zhang D, et al: miRNA-940 reduction contributes to human Tetralogy of Fallot development. J Cell Mol Med 18: 1830-1839, 2014.

17. Xu T, Zhou Q, Che L, Das S, Wang L, Jiang J, Li G, Xu J, Yao J, Wang $\mathrm{H}$, et al: Circulating miR-21, miR-378, and miR-940 increase in response to an acute exhaustive exercise in chronic heart failure patients. Oncotarget 7: 12414-12425, 2016.

18. Rajendiran S, Parwani AV, Hare RJ, Dasgupta S, Roby RK and Vishwanatha JK: MicroRNA-940 suppresses prostate cancer migration and invasion by regulating MIEN1. Mol Cancer 13: $250,2014$.

19. Ma J, Sun F, Li C, Zhang Y, Xiao W, Li Z, Pan Q, Zeng H, Xiao G, Yao K, et al: Depletion of intermediate filament protein nestin, a target of microRNA-940, suppresses tumorigenesis by inducing spontaneous DNA damage accumulation in human nasopharyngeal carcinoma. Cell Death Dis 5: e1377, 2014.

20. Ding D, Zhang Y, Yang R, Wang X, Ji G, Huo L, Shao Z and Li X: miR-940 Suppresses tumor cell invasion and migration via regulation of CXCR2 in hepatocellular carcinoma. Biomed Res Int 2016: 7618342, 2016.

21. Liu X, Ge X, Zhang Z, Zhang X, Chang J, Wu Z, Tang W, Gan L, Sun M and Li J: MicroRNA-940 promotes tumor cell invasion and metastasis by downregulating ZNF24 in gastric cancer. Oncotarget 6: 25418-25428, 2015.

22. Wang R, Wu Y, Huang W and Chen W: MicroRNA-940 targets INPP4A or GSK $3 \beta$ and activates the Wnt $/ \beta$-catenin pathway to regulate the malignant behavior of bladder cancer cells. Oncol Res 26: 145-155, 2018.

23. Yang HW, Liu GH, Liu YQ, Zhao HC, Yang Z, Zhao CL, Zhang XF and Ye H: Over-expression of microRNA-940 promotes cell proliferation by targeting GSK $3 \beta$ and sFRP1 in human pancreatic carcinoma. Biomed Pharmacother 83 593-601, 2016.

24. Yuan B, Liang Y, Wang D and Luo F: MiR-940 inhibits hepatocellular carcinoma growth and correlates with prognosis of hepatocellular carcinoma patients. Cancer Sci 106: 819-824, 2015.

This work is licensed under a Creative Commons Attribution-NonCommercial-NoDerivatives 4.0 International (CC BY-NC-ND 4.0) License. 